\title{
Avaliação de processo adsortivo utilizando mesocarpo de coco verde para remoção do corante azul de metileno
}

\section{Evaluation of the adsorption process using green coconut mesocarp for removal of methylene blue dye}

Fabiano Mendonça de Oliveira ${ }^{1}$, Luciana Melo Coelho ${ }^{1}$, Edmar Isaías de Melo

\footnotetext{
${ }^{1}$ Universidade Federal de Goiás, Unidade Acadêmica Especial de Física e Química, CEP 75704-020, Catalão, Goiás, Brasil.

${ }^{2}$ Universidade Federal de Uberlândia, Instituto de Química, CEP 38408-100, Uberlândia, Minas Gerais, Brasil. e-mail: mendonca_fabiano@hotmail.com, lucianacatalao@hotmail.com, emelo@iqufu.ufu.br
}

\section{RESUMO}

Os resíduos agroindustriais são materiais alternativos e de baixo custo, uma vez que quantidades consideráveis destes são descartadas no ambiente, com potencial uso como materiais adsorventes na remoção de poluentes, por exemplo. Nesse sentido, este trabalho foi realizado com o objetivo de avaliar a utilização da fibra de coco verde, como adsorvente na remoção do corante azul de metileno em meio aquoso. O corante azul de metileno é muito utilizado pela indústria têxtil e encontra-se presente em seus efluentes podendo contaminar o ambiente. A fibra de coco verde foi tratada com $\mathrm{HCl}, \mathrm{NaOH}$ e hexano. Os parâmetros avaliados foram $\mathrm{pH}$, tempo de agitação, mecanismo cinético e equilíbrio de adsorção. Os dados cinéticos foram ajustados aos modelos de Pseudo primeira ordem, Pseudo segunda ordem, Difusão intrapartícula e Quimissorção, resultando como o mais adequado para o processo o modelo de Pseudo segunda ordem. As isotermas de adsorção foram delineadas aos modelos de Langmuir e Freundlich, sendo este último o que apresentou melhor ajuste para os dados experimentais obtidos. A capacidade máxima adsortiva foi verificada, sendo o tratamento com $\mathrm{HCl}$ $\left(0,1 \mathrm{~mol} \mathrm{~L}^{-1}\right)$ aquele que proporcionou aumento significativo na capacidade adsortiva máxima $\left(166,7 \mathrm{mg} \mathrm{g}^{-1}\right)$ da fibra de coco verde quando comparada a fibra de coco in natura e aos demais tratamentos. Os resultados mostraram que a fibra de coco verde, tratada com ácido clorídrico, pode ser utilizada como material adsorvente na remediação de poluentes orgânicos, como corantes catiônicos em efluentes líquidos.

Palavras-chave: Adsorção; bioadsorvente; corante.

\begin{abstract}
Agroindustrial wastes are alternative and low cost materials, since considerable amounts of these are discarded in the environment, with potential use as adsorbent materials in the removal of pollutants, for example. In this sense, this work was carried out with the objective of evaluating the use of green coconut fiber as adsorbent in the removal of methylene blue dye in aqueous medium. The methylene blue dye is widely used by the textile industry and is present in its effluents and can contaminate the environment. The green coconut fiber was treated with $\mathrm{HCl}, \mathrm{NaOH}$ and hexane. The evaluated parameters were $\mathrm{pH}$, agitation time, kinetic mechanism and adsorption equilibrium. The kinetic data were adjusted to the Pseudo first order, Pseudo second order, Intraparticle diffusion and Quimisation models, resulting as the most suitable for the Pseudo second order model. The adsorption isotherms were delineated to the Langmuir and Freundlich models, the latter being the best fit for the experimental data obtained. The maximum adsorptive capacity was verified by treatment with $\mathrm{HCl}\left(0.1 \mathrm{~mol} \mathrm{~L}^{-1}\right)$ that provided a significant increase in the maximum adsorptive capacity $\left(166.67 \mathrm{mg} \mathrm{g}^{-1}\right)$ of green coconut fiber compared to coconut fiber in and other treatments. The results showed that green coconut fiber, treated with hydrochloric acid, can be used as adsorbent material in the remediation of organic pollutants such as cationic dyes in liquid effluents.
\end{abstract}

Keywords: Adsorption; bioadsorbent; dye. 


\section{INTRODUÇÃO}

Nas últimas décadas, os problemas ambientais têm se tornado cada vez mais intensos e frequentes, principalmente devido ao elevado crescimento populacional e ao aumento da atividade industrial. Problemas ocasionados pela interferência do ser humano têm provocado impactos irreversíveis na qualidade do solo, ar e água. Nesse contexto, o setor têxtil apresenta especial destaque devido a elevada quantidade de efluente gerado, os quais, quando não tratados corretamente, podem causar sérios problemas de contaminação ambiental [1].

Considerando o volume descartado e a diversidade na composição, o efluente da indústria têxtil é classificado como o mais poluente de todos os setores da indústria química, uma vez que, no Brasil, a indústria têxtil utiliza aproximadamente $20 \mathrm{t}_{\text {ano }}{ }^{-1}$ de corantes e cerca de $20 \%$ são descartados como efluentes; o principal motivo desta perda está relacionado à incompleta fixação do corante à fibra do tecido, durante o processo de tingimento. Se o efluente não for tratado adequadamente antes de ser descartado, os corantes podem provocar danos graves ao ecossistema e à saúde da população [2].

Entre os corantes de maior aplicabilidade destaca-se a classe dos reativos, dentre eles o azul de metileno um corante catiônico do grupo das fenotiazinas, muito empregado na indústria têxtil no tingimento de tecidos de algodões e lãs, gerando efluente que afeta não só a transparência das águas, mas também limita a passagem de radiação solar diminuindo a atividade fotossintética natural provocando alterações na biota aquática, originando toxicidade aguda e crônica de ecossistemas aquáticos [3].

Os métodos convencionais para descontaminação de águas provenientes da indústria têxtil contendo corantes são economicamente desfavoráveis e/ou tecnicamente complexos [4]. Assim, o processo de adsorção surge como alternativa para a remoção de corantes, utilizando materiais adsorventes, que apresentem baixo custo, disponibilidade, elevada capacidade e taxa de adsorção [5].

Nesse sentido, abre-se espaço para pesquisas de novos materiais adsorventes, que possibilitem a descontaminação de efluentes pelo processo de adsorção. Nos últimos anos a pesquisa sobre a utilização de resíduos agroindustriais para a remediação de ambientes contaminados pelo processo de adsorção vem ganhando destaque devido à elevada quantidade de resíduos que a agroindústria produz que por sua vez podem constituir sérios problemas de disposição final, como é o caso da casca de coco verde, pois cerca de 80 a $85 \%$ do seu peso bruto representa resíduo, levando este até oito anos para se decompor [6,7].

Fundamentando-se nos pressupostos apontados anteriormente, os resíduos provenientes da agroindústria, dentre eles a casca de coco verde e sua aplicação como adsorvente utilizado na remoção de contaminantes pode ser uma alternativa promissora para remediação de efluentes pelo processo de adsorção, dado à sua disponibilidade, caráter de produto renovável, exigência de pouco preparo e baixo custo para sua utilização. Considerando as características e vantagens da fibra de coco verde, aliada as vantagens do processo de adsorção para remediação de efluentes, o presente trabalho objetivou avaliar a fibra do coco verde in natura e tratada, como material adsorvente alternativo, aplicado na remoção do corante azul de metileno em meio aquoso por adsorção.

\section{MATERIAIS E MÉTODOS}

\subsection{Adsorvato}

O corante azul de metileno (C.I. 52030, $\mathrm{C}_{16} \mathrm{H}_{18} \mathrm{~N}_{3} \mathrm{SCl}$ ) utilizado neste estudo foi obtido da Sigma Chemical, USA, com grau analítico e usado sem purificação suplementar.

\subsection{Preparação e caracterização do adsorvente}

O coco verde foi obtido de comércio local em Monte Carmelo-MG. O coco verde foi cortado em pedaços, separando-se o mesocarpo do endocarpo; em seguida o mesocarpo foi triturado e lavado com água deionizada e seco em estufa com circulação de ar a $45^{\circ} \mathrm{C}$ até massa constante. A separação granulométrica das fibras in natura foi realizada em peneira com malha de $0,25 \mathrm{~mm}$. Parte deste material com diâmetro de partícula menor que $0,25 \mathrm{~mm}$, foi tratado com $50,0 \mathrm{~mL}$ com solução $\mathrm{HCl} 0,1 \mathrm{~mol} \mathrm{~L}^{-1}$, submetido a agitação por uma hora, filtrado e lavado com água deionizada a fim de eliminar o excesso de $\mathrm{HCl}$, até que o valor do $\mathrm{pH}$ do filtrado ficasse igual a 7,00. A fibra de coco verde in natura também foi tratada com $\mathrm{NaOH} 0,1 \mathrm{~mol} \mathrm{~L}^{-1} \mathrm{e}$ hexano, utilizando o mesmo procedimento. A caracterização dos materiais adsorventes foi realizada por espectrometria no Infravermelho, empregando um Espectrofotômetro de Infravermelho por transformada de Fourier FTIR Prestige-21, Shimadzu. Os espectros foram registrados a partir de 4000 a $500 \mathrm{~cm}^{-1}$. A morfologia superficial foi avaliada utilizando um Microscópio Eletrônico de Varredura JSM-6610. 


\subsection{Ensaios de adsorção}

Os ensaios de adsorção foram realizados adicionando $10,0 \mathrm{mg}$ do material adsorvente (diâmetro médio de partícula menor que $0,25 \mathrm{~mm}) \mathrm{em} 25,0 \mathrm{~mL}$ de solução do corante $\left(6 \mathrm{mg} \mathrm{L}^{-1}\right)$ mantido sob agitação por 20 min à temperatura ambiente. Após esse período foi realizada a separação da biomassa por centrifugação durante 5 minutos a 3.000 rpm e a concentração final do corante em solução foi determinada utilizando um espectrofotômetro de UV-VIS (HACH DR/4000) a 665nm, com limite de detecção, 0,04 $\mathrm{mg} \mathrm{L}^{-1}$ e limite de quantificação de $0,12 \mathrm{mg} \mathrm{L}^{-1}$. A quantidade de corante adsorvida por massa do adsorvente no equilíbrio (capacidade adsortiva $\mathrm{q}_{\mathrm{e}}$ ) foi calculada utilizando a Equação 1.

$$
q_{e}=\frac{\left(C_{0}-C_{e}\right) V_{L}}{m_{a d s}}
$$

Sendo: $\mathrm{q}_{\mathrm{e}}=$ quantidade de corante adsorvida dada em mg do adsorvato $\mathrm{g}^{-1}$ do adsorvente; $\mathrm{C}_{0}$ e $\mathrm{C}_{\mathrm{e}}$ são as concentrações em fase líquida iniciais e no equilíbrio, respectivamente $\left(\mathrm{mg} \mathrm{L}^{-1}\right) ; \mathrm{V}_{\mathrm{L}}=$ volume da solução (L) e $\mathrm{m}_{\mathrm{ads}}$ é massa de adsorvente $(\mathrm{g})$.

\subsection{Efeito do pH e avaliação do pH do ponto de carga zero}

$\mathrm{O}$ efeito do $\mathrm{pH}$ foi investigado em soluções do corante azul de metileno em diferentes níveis de pH (2, 4, 6, 7, 8,10 e 12) por meio de ensaios de adsorção (10,0 mg de material adsorvente e $25,0 \mathrm{~mL}$ de solução de azul e metileno, $6,0 \mathrm{mg} \mathrm{L}^{-1}$, tempo de agitação 20 minutos). $\mathrm{O}$ valor do $\mathrm{pH}$ inicial foi ajustado com soluções de $\mathrm{HCl}$ $\left(0,1 \mathrm{~mol} \mathrm{~L}^{-1}\right)$ e $\mathrm{NaOH}\left(0,1 \mathrm{~mol} \mathrm{~L}^{-1}\right)$ utilizando medidor de $\mathrm{pH}$, Modelo HI 2223-01, marca Hanna.

A avaliação do $\mathrm{pH}$ do ponto de carga zero $\left(\mathrm{pH}_{\mathrm{PCZ}}\right)$ foi realizada em duplicata adicionando $20 \mathrm{mg}$ dos materiais adsorventes em $20,0 \mathrm{~mL}$ de solução aquosa de $\mathrm{KCl} 0,1 \mathrm{~mol} \mathrm{~L}^{-1}$ em diferentes níveis de $\mathrm{pH}$ inicial (1, $2,3,4,5,6,7,8,9,10,11,12)$, ajustado com soluções de $\mathrm{HCl}\left(0,1 \mathrm{~mol} \mathrm{~L}^{-1}\right)$ e $\mathrm{NaOH}\left(0,1 \mathrm{~mol} \mathrm{~L}^{-1}\right)$. Após $24 \mathrm{~h}$ de agitação, em temperatura ambiente, as soluções foram filtradas e o pH final da solução foi avaliado.

\subsection{Estudo cinético}

Ensaios de adsorção utilizando $10,0 \mathrm{mg}$ de material adsorvente e $25,0 \mathrm{~mL}$ de solução de azul de metileno (6 $\mathrm{mg} \mathrm{L}^{-1}$ ) e pH inicial igual 8,00 foram realizados em diferentes tempos de agitação (5, 20, 40, 60 e 120 minutos). Para avaliar o processo cinético de adsorção, foram aplicados os modelos de Pseudo primeira ordem, Pseudo segunda ordem, Difusão intrapartícula e Quimiossorção [8, 9 , 10, 11].

\subsection{Isotermas de adsorção}

Ensaios de adsorção em triplicata foram realizados com 50,0 mL de solução em diferentes concentrações de azul de metileno $\left(6,0 ; 12,0 ; 18,0 ; 24,0 ; 28,0 ; 32,0 ; 36,0 ; 40,0 ; 44,0\right.$ e $\left.48,0 \mathrm{mg} \mathrm{L}^{-1}\right)$, pH inicial igual a 8 . O tempo de agitação e a massa do material adsorvente foram 60 minutos e $10 \mathrm{mg}$, respectivamente. O tempo de agitação 40 minutos e massa de adsorvente $20 \mathrm{mg}$ foram utilizados para o material tratado com hexano. Foram calculados os valores de $\mathrm{C}_{\mathrm{e}}$, quantidade de soluto adsorvido na fase fluida $\left(\mathrm{mg} \mathrm{L}^{-1}\right)$ e $\mathrm{q}_{\mathrm{e}}$, quantidade de soluto adsorvido na fase sólida $\left(\mathrm{mg} \mathrm{g}^{-1}\right)$ e os modelos de Langmuir e Freundlich [11] foram utilizados na avaliação do equilíbrio de adsorção.

\section{RESULTADOS E DISCUSSÃO}

\subsection{Caracterização do material adsorvente}

Os espectros no infravermelho para a fibra de coco verde in natura e tratada, Figura 1, apresentam uma banda intensa em $3408 \mathrm{~cm}^{-1}$ que é atribuída às vibrações de estiramento, da ligação $(\mathrm{OH})$, pertencentes à estrutura da celulose e hemicelulose, que se constitui no principal componente dos materiais lignocelulósico [12]. As bandas ocasionadas pelos estiramentos em aproximadamente $2927 \mathrm{~cm}^{-1},(\mathrm{C}-\mathrm{H})$, e bandas em $1326 \mathrm{~cm}^{-1}$ são atribuídas às vibrações do grupo $-\mathrm{CH}_{2}$ presentes na celulose e hemicelulose [13]. A presença das bandas em 1514,1449 e $1610 \mathrm{~cm}^{-1}$ são atribuídas aos estiramentos $(\mathrm{C}=\mathrm{C})$ de grupos aromáticos isolados da lignina [14]. As vibrações na região de $1736 \mathrm{~cm}^{-1}$ correspondem ao estiramento da carbonila [15]. A banda em 1250 $\mathrm{cm}^{-1}$ é atribuída às vibrações $(\mathrm{C}-\mathrm{O})$ de ésteres, éteres, ou grupos fenóis. A banda em $1040 \mathrm{~cm}^{-1}$ é designada para $(\mathrm{C}-\mathrm{O})$ de álcoois primários. A banda de absorção em $895 \mathrm{~cm}^{-1}$ e característica das ligações glicosídicas $\beta(1 \rightarrow 4)$; a banda presente em $823 \mathrm{~cm}^{-1}$ é indicativa da presença de unidades de p-hidroxifenil, um dos precursores da lignina, e as bandas localizadas em $1167 \mathrm{~cm}^{-1}$ derivam do estiramento do grupo C-O-C de ligações glicosídicas $\beta(1 \rightarrow 4)$ [16]. Desta forma é possível observar que os principais grupos oxigenados pre- 
sentes na fibra de coco verde in natura e tratada estão distribuídos na forma de ésteres, éteres, álcoois e fenóis. Esses grupos podem estar associados ao processo de adsorção pelo material adsorvente.

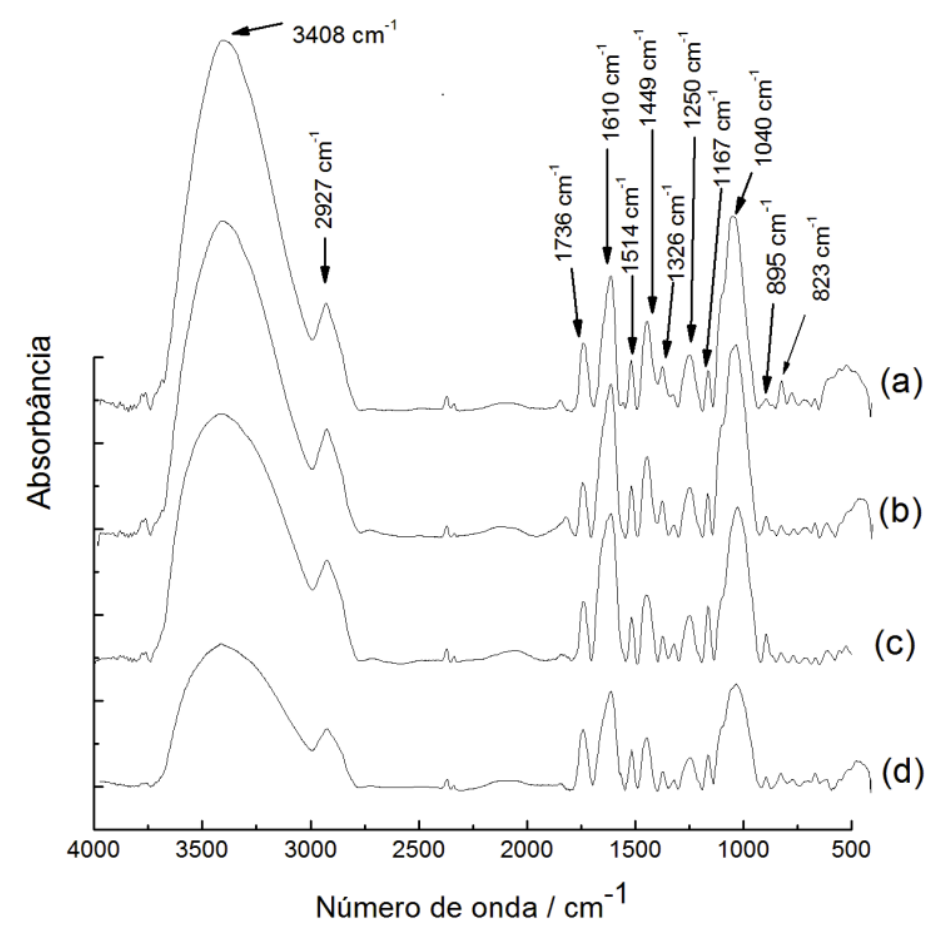

Figura 1: Espectros no infravermelho da fibra de coco verde. In natura (a); tratado com $\mathrm{HC} \ell$ 0,1 $\mathrm{mol} \mathrm{L}^{-1}$ (b); tratado com $\mathrm{NaOH} 0,1 \mathrm{~mol} \mathrm{~L}^{-1}$ (c) tratado com hexano (d).

As características morfológicas da superfície dos materiais adsorventes (Figura 2, 3,4 e 5) evidenciam diferenças entre a morfologia da superfície das fibras não tratadas e tratadas. As imagens apresentadas na figura 2 mostram a existência de irregularidades na superfície da fibra de coco in natura cujas camadas de cutículas revestem as fibras com uma cobertura em formato de couraça. Essas irregularidades são "promoções" externas (cutículas), isto é, cera de origem alifática $[17,18]$ e outros constituintes, provavelmente resíduos orgânicos $[19,20]$, que se instalam na superfície das fibras e propiciam uma morfologia irregular. No tratamento com $\mathrm{HCl}$ (Figura 3), também são observadas camadas de cutículas que revestem a fibra com uma cobertura em formato de couraça, originando uma morfologia irregular.

As imagens de microscopia eletrônica de varredura da fibra tratada com $\mathrm{NaOH}$ (Figura 4), apresentam morfologia lisa e regular, provavelmente devido a remoção de impurezas e das camadas de cutículas (cera de origem alifática). As fibrilas apresentam-se separadas umas das outras, provavelmente devido a remoção da lignina e hemicelulose em meio alcalino ou por prováveis mudanças nas interações de ligação de hidrogênio de grupos hidroxílicos de celulose, resultando na deformação de microfibrilas individuais o que aumentou a irregularidade da superfície, visualizado na forma de cavidades [21].

A fibra tratada com hexano (Figura 5), apresenta superfície mais regular em relação a fibra in natura. $\mathrm{O}$ tratamento com hexano promoveu a retirada de parte da camada de cutícula da fibra de coco, formando uma morfologia mais lisa e regular, porém observa-se a presença de partículas globulares fixadas em cavidades específicas da própria fibra.

Avaliando o diâmetro médio da fibra de coco in natura $(121 \mu \mathrm{m})$ e fibra de coco tratadas $(124 \mu \mathrm{m}$, $80 \mu \mathrm{m}$ e $93 \mu \mathrm{m}$ para os tratamentos com $\mathrm{HCl}, \mathrm{NaOH}$ e hexano, respectivamente), verifica-se que o tratamento alcalino, apresentou menor diâmetro médio em relação aos demais tratamentos, o que pode ser devido a remoção da lignina, hemicelulose e impurezas presentes na superfície das fibras. Comportamento semelhante referente a diminuição do diâmetro médio das fibras, após tratamento com $\mathrm{NaOH}$, também foi observado por ESMERALDO [19]. 

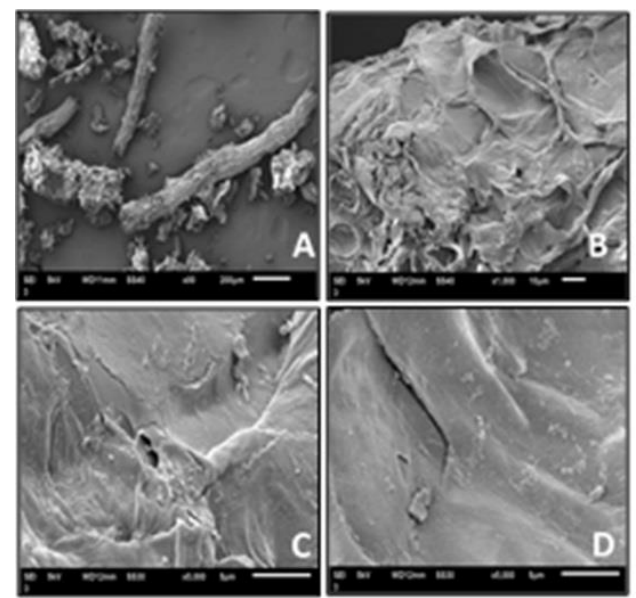

Figura 2: Fotomicrografias eletrônicas de varredura para a amostra da fibra de coco verde in natura. Aumento: 80X (A); 1000X (B); 5000X (C) e (D).

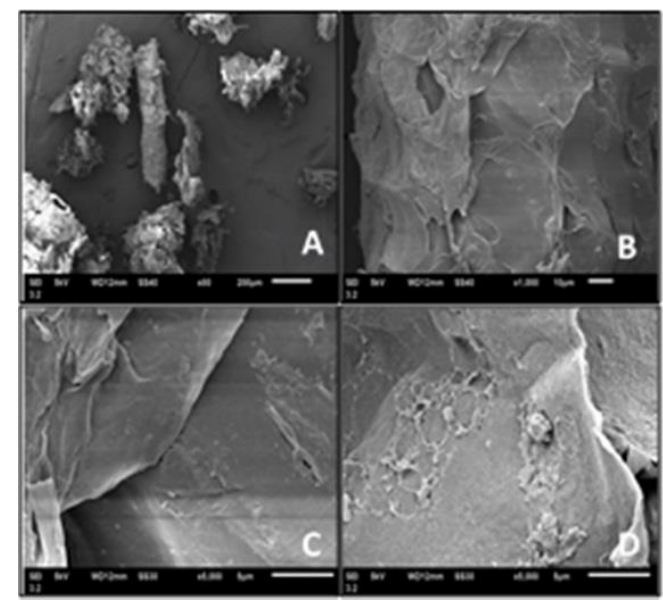

Figura 3: Fotomicrografias eletrônicas de varredura para fibra de coco verde tratada com $\mathrm{HCl} 0,1 \mathrm{~mol} \mathrm{~L}^{-1}$. Aumento: 80X (A); 1000X (B); 5000X (C) e (D).

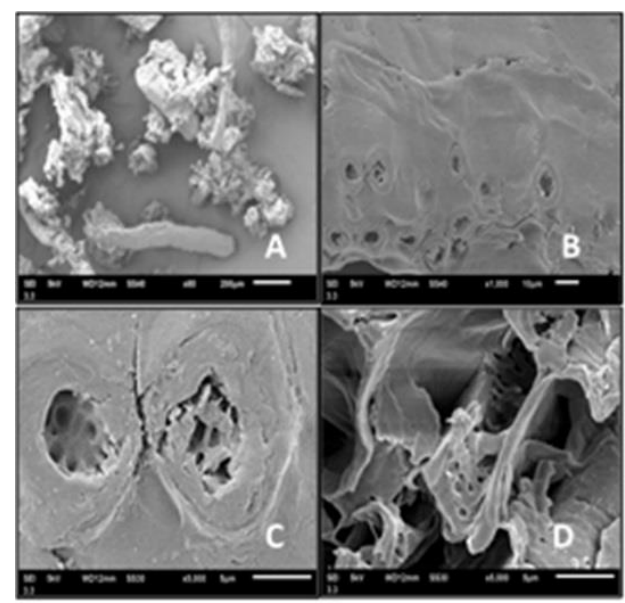

Figura 4: Fotomicrografias eletrônicas de varredura para fibra de coco verde tratada com $\mathrm{NaOH} 0,1 \mathrm{~mol} \mathrm{~L}^{-1}$. Aumento: 80X (A); 1000X (B); 5000X (C) e (D). 


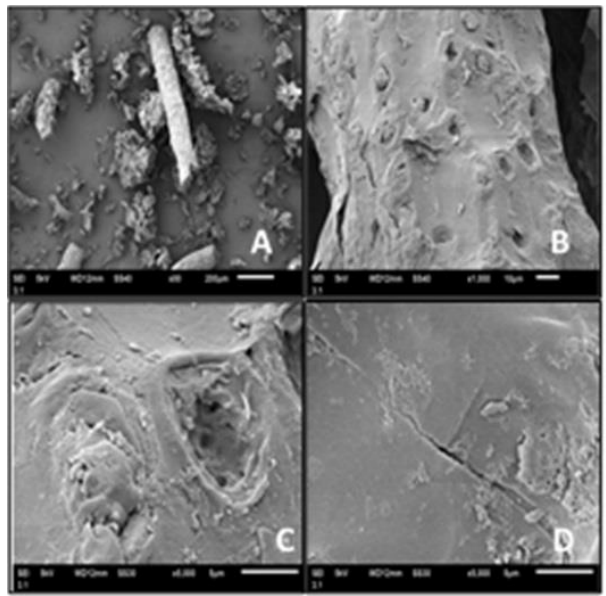

Figura 5: Fotomicrografias eletrônicas de varredura para fibra de coco verde tratada com hexano. Aumento: 80X (A); 1000X (B); 5000X (C) e (D).

\subsection{Estudo de pH}

Foram realizados testes variando-se o $\mathrm{pH}$ inicial das soluções para verificar o efeito deste parâmetro na adsorção do corante, uma vez que, o pH da solução pode modificar a carga superficial do adsorvente, assim como influenciar no grau de ionização da molécula de adsorvato e no grau de dissociação de grupos funcionais sobre os sítios ativos do adsorvente [22]. A avaliação do efeito do $\mathrm{pH}$ na capacidade adsortiva da fibra de coco verde in natura e tratada está apresentada na Figura 6.

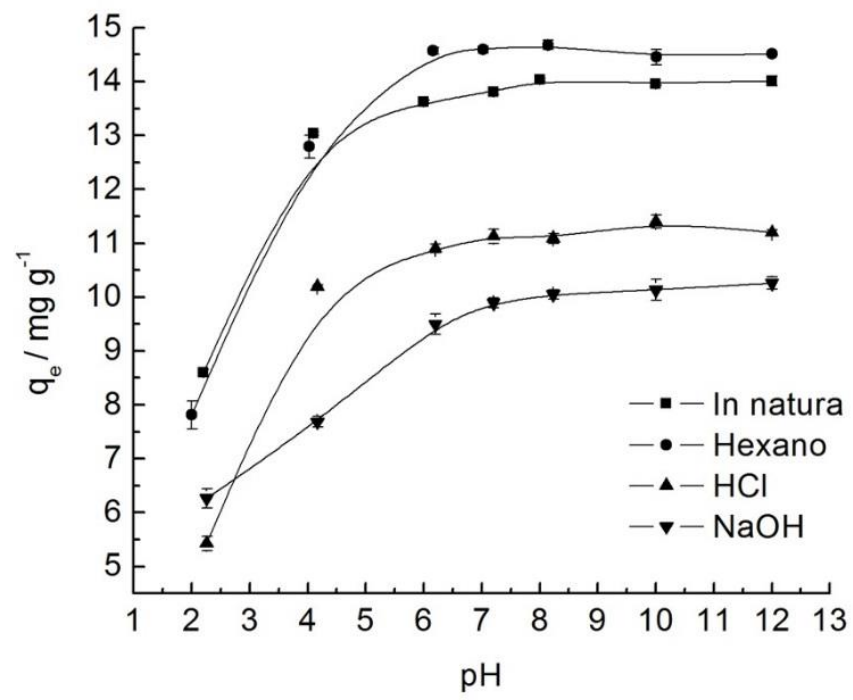

Figura 6: Capacidade adsortiva da fibra de coco verde in natura e tratada em função do pH da solução de azul de metileno.

A capacidade adsortiva permaneceu constante num intervalo de $\mathrm{pH}$ entre 7 e 12 . Os menores valores de capacidade adsortiva foram verificados em pH 2. A adsorção aumenta com o aumento do $\mathrm{pH}$, sendo o equilíbrio de adsorção estabelecido a partir de $\mathrm{pH}$ 7. A baixa capacidade adsortiva em meio ácido é devido provavelmente à presença de íons $\mathrm{H}^{+}$em excesso que entram em competição com os grupos catiônicos do corante e os sítios de adsorção. Em meio alcalino, a superfície do material adsorvente pode apresentar carga superficial líquida negativa, conforme verificado na avaliação do $\mathrm{pH}_{\mathrm{PCZ}}$ (Figura 7), o que favorece o processo de adsorção do corante catiônico. A capacidade adsortiva mais acentuada foi verificada para o tratamento com hexano $\left(13,81 \mathrm{mg} \mathrm{g}^{-1}\right)$. 

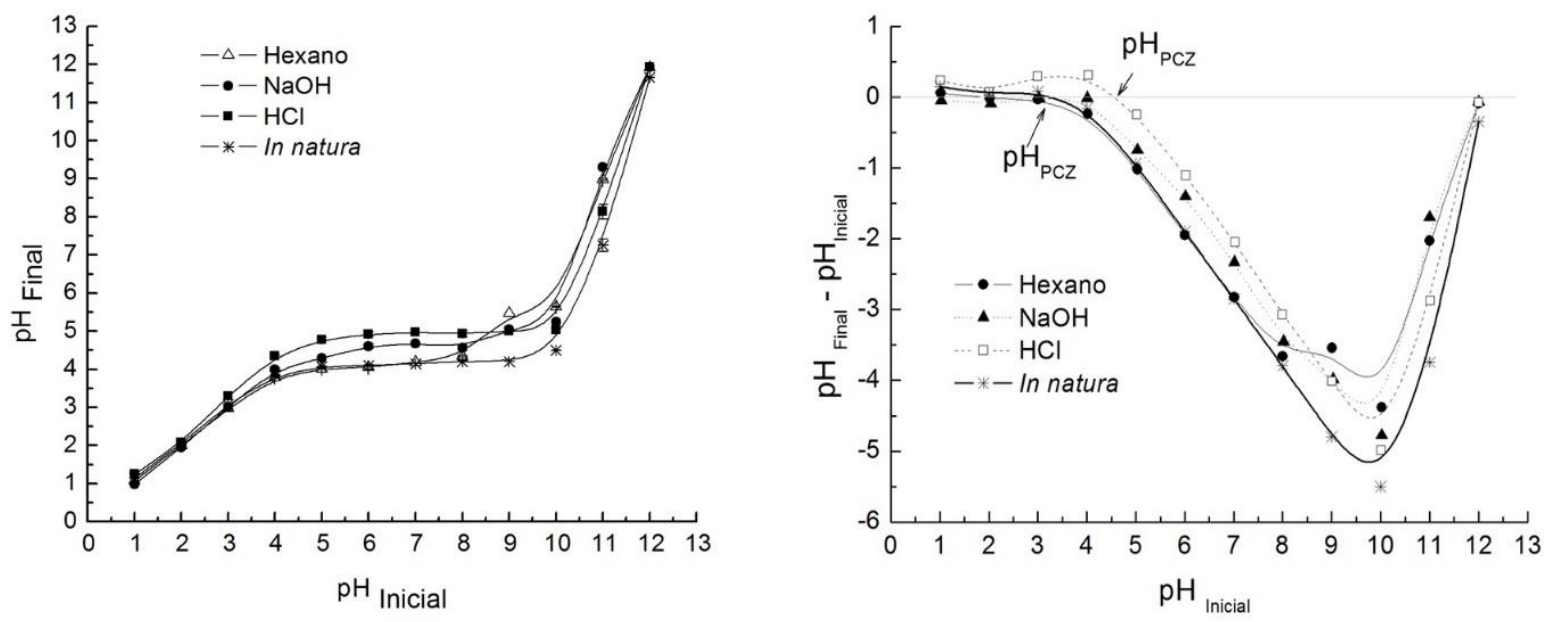

Figura 7: Ponto de carga zero da fibra de coco verde in natura e tratada.

\subsection{Estudo cinético}

O estudo da cinética de adsorção é um importante parâmetro utilizado em estudos relacionados ao tratamento de efluentes aquosos, pois fornece informações sobre processos de adsorção tais como: o tempo requerido para cada equilíbrio, velocidades com quais as moléculas do adsorvato são adsorvidas pelo adsorvente [23].

A evolução da cinética de adsorção do corante azul de metileno para a fase sólida, utilizando como material adsorvente a fibra de coco verde in natura e tratada pode ser visualizada na Figura 8.

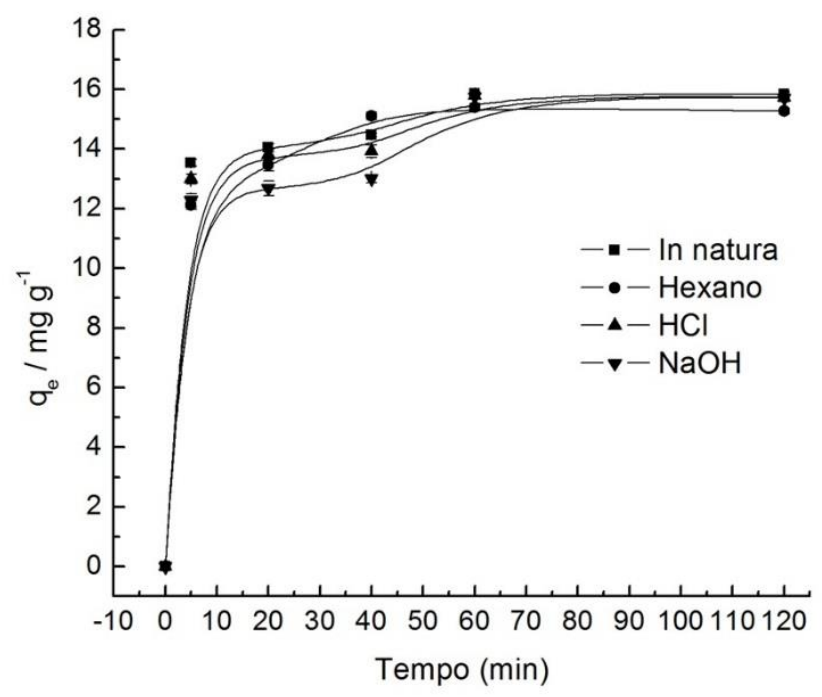

Figura 8: Efeito do tempo de agitação na remoção do corante azul de metileno para fibra de coco verde in natura e tratada.

Vários modelos cinéticos são utilizados para examinar o mecanismo controlador do processo de adsorção, tais como, reação química, controle da difusão e transferência de massa. Os modelos empregados com maior frequência são os de Pseudo primeira ordem e de Pseudo segunda ordem [8]. Contudo, o mecanismo do processo de adsorção pode não ser obtido por estes dois modelos, sendo necessário a aplicação de outros modelos, dentre eles o modelo da Difusão intrapartícula de Weber e Morris e o modelo cinético de Quimiossorção de Elovich, por exemplo.

A velocidade de adsorção pode ser determinada por expressão da velocidade de Pseudo primeira ordem dada por Lagergren para a adsorção em sistema líquido/sólido baseada na capacidade do sólido. A velocidade de remoção do adsorvato com o tempo é diretamente proporcional à diferença na concentração de 
saturação e ao número de sítios ativos do sólido. A equação cinética de Lagergren é a mais usada para a adsorção de um adsorvato de uma solução aquosa [8].

Uma análise simples da cinética de adsorção realizada pela Equação de Lagergren, de Pseudo primeira ordem, baseada na capacidade dos sólidos é dada pela Equação 2:

$$
\frac{d q_{\mathrm{t}}}{d t}=k_{1}\left(q_{e}-q_{t}\right)
$$

Sendo: $\mathrm{k}_{1}$ é a constante da taxa de adsorção de Pseudo primeira ordem $\left(\mathrm{min}^{-1}\right)$, e $\mathrm{q}_{\mathrm{e}}$ e $\mathrm{q}_{\mathrm{t}}$ são as quantidades adsorvidas por grama de adsorvente no equilíbrio e no tempo $\mathrm{t}$, respectivamente $\left(\mathrm{mg} \mathrm{g}^{-1}\right)$.

Após a integração da Equação 2 e aplicando-se condições de contorno: $\mathrm{t}=0$ a $\mathrm{t}=\mathrm{t}$ e $\mathrm{q}_{\mathrm{t}}=$ $0, \mathrm{q}_{\mathrm{t}}=\mathrm{q}_{\mathrm{t}}$, obtém-se a Equação 3 que pode ser expressa pela Equação 4 na sua forma linear.

$$
\begin{aligned}
& \ln \left(\frac{q_{e}}{q_{e}-q_{t}}\right)=k_{1} t \\
& \ln \left(q_{e}-q_{t}\right)=\ln q_{e}-k_{1}
\end{aligned}
$$

$\mathrm{O}$ valor de $\mathrm{k}_{1}$ pode ser determinado através do gráfico de $\ln \left(\mathrm{q}_{\mathrm{e}}-\mathrm{q}_{\mathrm{t}}\right)$ versus $\mathrm{t}$. $\mathrm{O}$ ajuste da equação aos dados experimentais exige que a capacidade de adsorção no equilíbrio, $\mathrm{q}_{\mathrm{e}}$, seja conhecida [9].

Os dados cinéticos foram também analisados usando as cinéticas de Pseudo segunda ordem, onde a velocidade da reação é dependente da quantidade do soluto adsorvido na superfície do adsorvente e da quantidade adsorvida no equilíbrio. O modelo linear de Pseudo segunda ordem pode ser expresso de acordo com a equação 5:

$$
\frac{d q_{\mathrm{t}}}{d_{\mathrm{t}}}=k_{2}\left(q_{e}-q_{t}\right)^{2}
$$

Sendo: $\mathrm{k}_{2}=$ constante da taxa de adsorção de pseudo segunda ordem $\left(\mathrm{g} \mathrm{mg}^{-1} \mathrm{~min}^{-1}\right)$.

Integrando a Equação 5, similarmente, à Equação 2 obtém-se a Equação 6.

$\frac{t}{\left(q_{e}-q_{t}\right)}=\frac{1}{q_{t}^{2}}+k_{2} t$

Linearizando a Equação 6, tem-se a Equação 7:

$$
\frac{t}{q_{\mathrm{t}}}=\frac{1}{k_{2} q_{e}^{2}}+\frac{t}{q_{e}}
$$

Os valores de $\mathrm{q}_{\mathrm{e}}$ e $\mathrm{k}_{2}$ podem ser obtidos através do intercepto e da inclinação da curva apresentada no gráfico $\left(\mathrm{t} / \mathrm{q}_{\mathrm{t}}\right)$ versus t. Se o modelo cinético de Pseudo segunda ordem for aplicável, a plotagem de $\left(\mathrm{t} / \mathrm{q}_{\mathrm{t}}\right)$ versus t deve apresentar relação linear próxima a 1 [9].

De acordo com WEBER e MORRIS [10,11], se a difusão intrapartícula é o fator determinante da velocidade, a remoção do adsorvato varia com a raiz quadrada do tempo. Assim, o coeficiente de difusão intrapartícula $\left(\mathrm{k}_{\mathrm{dif}}\right)$ pode ser definido pela equação 8 .

$$
q_{\mathrm{t}}=k_{\text {dif }} t^{\frac{1}{2}}+C
$$

Sendo: $\mathrm{q}_{\mathrm{t}}=\mathrm{a}$ quantidade de corante adsorvida $\left(\mathrm{mg} \mathrm{g}^{-1}\right) ; \mathrm{t}=\mathrm{o}$ tempo de agitação $(\mathrm{min}) ; \mathrm{C}\left(\mathrm{mg} \mathrm{g}^{-1}\right)=$ constante relacionada com a resistência à difusão.

$\mathrm{O}$ valor de $\mathrm{k}_{\mathrm{dif}}\left(\mathrm{mg} \mathrm{g}^{-1} \min ^{-1 / 2}\right)$ pode ser obtido da inclinação e o valor de $\mathrm{C}$ da intersecção da curva do gráfico $\mathrm{q}_{\mathrm{t}}$ versus $\mathrm{t}^{1 / 2}$. Os valores de $\mathrm{C}$ dão uma ideia da espessura da camada limite, isto é, quanto maior for $\mathrm{o}$ valor de $\mathrm{C}$ maior será o efeito da camada limite [9].

A equação de Elovich é aplicada para a cinética de quimiossorção. Essa equação tem sido aplicada satisfatoriamente em alguns processos de quimiossorção e tem sido aplicada com sucesso em processos de cinética de adsorção lenta [24]. A equação cinética é válida para sistemas nos quais a superfície do adsorvente é heterogênea e é formulada como:

$$
\frac{d q_{t}}{d t}=\alpha \exp \left(-\beta q_{\mathrm{t}}\right)
$$

Integrando essa equação nas condições de contorno, $\mathrm{t}=0$ a $\mathrm{t}=\mathrm{t}$ e $\mathrm{q}_{\mathrm{t}}=0$ a $\mathrm{q}_{\mathrm{t}}=\mathrm{q}_{\mathrm{t}}$, origina-se: 


$$
q_{t}=\frac{1}{\beta} \ln \left(t+t_{0}\right)-\frac{1}{\beta} \ln \left(t_{0}\right)
$$

Sendo: $\alpha=$ a taxa inicial de adsorção $\left(\mathrm{mg} \mathrm{g}^{-1} \min ^{-1}\right) ; \beta=$ constante relacionada ao grau de cobertura e a energia de ativação envolvida no processo de quimissorção $\left(\mathrm{g} \mathrm{mg}^{-1}\right) ; \mathrm{t}_{0}=1 / \alpha \beta$. a seguir:

Se $t$ for muito maior que $t_{0}$ [24], a equação 10 pode ser simplificada e reescrita conforme a equação 11

$$
q_{t}=\frac{1}{\beta} \ln (\alpha \cdot \beta)+\frac{1}{\beta} \ln (t)
$$

Para avaliar a cinética de adsorção, os dados experimentais descritos na figura 8 , foram ajustados aos modelos de Pseudo primeira ordem, Pseudo segunda ordem, Difusão intrapartícula de Weber e Morris e Quimissorção de Elovich, estando os parâmetros cinéticos do ajuste destes modelos apresentados na Tabela 1.

Tabela 1: Parâmetros cinéticos para a remoção do azul de metileno utilizando fibra de coco verde in natura e tratada

\begin{tabular}{|c|c|c|c|c|c|}
\hline & & & & \\
\hline & & in natura & hexano & $\mathrm{HCl}$ & $\mathrm{NaOH}$ \\
\hline \multirow{4}{*}{$\begin{array}{c}\text { Pseudo } \\
\text { primeira ordem }\end{array}$} & $\mathrm{K}_{\mathrm{f}}\left(\min ^{-1}\right)$ & 0,015499 & 0,032795 & 0,039934 & 0,040671 \\
\hline & $\mathrm{q}_{\mathrm{e}}\left(\mathrm{mg} \mathrm{g}^{-1}\right)$ & 3,989973 & 4,739254 & 7,20228 & 9,032126 \\
\hline & $\mathrm{R}^{2}$ & 0,66779 & 0,85479 & 0,95235 & 0,96576 \\
\hline & $F_{\text {error }}$ & 0,37546 & 0,4326 & 0,27801 & 0,23748 \\
\hline \multirow{5}{*}{$\begin{array}{c}\text { Pseudo } \\
\text { segunda ordem }\end{array}$} & $\mathrm{K}_{\mathrm{s}}\left(\mathrm{g} \mathrm{mg}^{-1} \min ^{-1}\right)$ & 0,03945 & 0,057789 & 0,032581 & 0,020215 \\
\hline & $\mathrm{q}_{\mathrm{e}}\left(\mathrm{mg} \mathrm{g}^{-1}\right)$ & 15,99488 & 15,44402 & 15,43686 & 15,48947 \\
\hline & $\mathrm{h}_{0}\left(\mathrm{mg} \mathrm{g}^{-1} \min ^{-1}\right)$ & 10,09285 & 13,7836 & 7,763975 & 4,850131 \\
\hline & $\mathrm{R}^{2}$ & 0,99937 & 0,99972 & 0,99881 & 0,99684 \\
\hline & $\mathrm{F}_{\text {error }}$ & 0,11115 & 0,07685 & 0,15803 & 0,25737 \\
\hline \multirow{4}{*}{ Quimissorçãa } & $\alpha\left(\mathrm{mg} \mathrm{g}^{-1} \min ^{-1}\right)$ & 2791949 & 8,81284 & 9,232772 & 7,902724 \\
\hline & $\beta\left(\mathrm{g} \mathrm{mg}^{-1}\right)$ & 1,254453 & 0,3306 & 0,345105 & 0,346021 \\
\hline & $\mathrm{R}^{2}$ & 0,91282 & 0,89793 & 0,88232 & 0,90039 \\
\hline & $\mathrm{F}_{\text {error }}$ & 0,49816 & 2,93634 & 3,06108 & 2,76557 \\
\hline \multirow{4}{*}{$\begin{array}{c}\text { Difusão intra- } \\
\text { partícula }\end{array}$} & $\mathrm{K}_{\mathrm{dif}}\left(\mathrm{mg} \mathrm{g}^{-1} \min ^{-1 / 2}\right)$ & 1,17865 & 1,19239 & 1,15277 & 1,17706 \\
\hline & $\mathrm{C}$ & 6,05961 & 5,58749 & 5,5565 & 4,95527 \\
\hline & $\mathrm{R}^{2}$ & 0,75956 & 0,78505 & 0,77849 & 0,81333 \\
\hline & $F_{\text {error }}$ & 4,43321 & 4,13236 & 4,08219 & 3,69819 \\
\hline
\end{tabular}
como adsorvente. Condições experimentais: $\mathrm{m} / \mathrm{v}$ : Fibra de coco verde $=0,4 \mathrm{mg} \mathrm{L}^{-1} ; \mathrm{pH}=8$ e $\mathrm{C}_{0}=6 \mathrm{mg} \mathrm{L}^{-1}$.

O modelo cinético de Pseudo segunda ordem foi o que apresentou melhor ajuste em relação aos demais modelos (menor $\mathrm{F}_{\text {error }}$ ), indicando uma menor diferença entre o valor de $\mathrm{q}_{\mathrm{e}}$ calculado e aquele obtido experimentalmente $[25,26]$.

Os valores de $\mathrm{q}_{\mathrm{e}}$ calculados segundo este modelo $\left(15,99 ; 15,44 ; 15,43\right.$ e $15,48 \mathrm{mg} \mathrm{g}^{-1}$, in natura, hexano, $\mathrm{HCl}$ e $\mathrm{NaOH}$, respectivamente) apresentaram boa concordância com os valores de $\mathrm{q}_{\mathrm{e}}$ experimental $\left(15,85 ; 15,26 ; 15,29\right.$ e $15,26 \mathrm{mg} \mathrm{g}^{-1}$, in natura, hexano, $\mathrm{HCl} \mathrm{e} \mathrm{NaOH}$, respectivamente).

\subsection{Equilíbrio de adsorção}

O comportamento da adsorção pode ser avaliado quantitativamente através das isotermas de adsorção. Elas expressam a relação entre a quantidade do corante que é adsorvido por unidade de massa do adsorvente e a concentração do corante em solução no equilíbrio a temperatura constante. A expressão gráfica da isoterma é geralmente uma hipérbole. A adsorção pode ser avaliada quantitativamente através das isotermas. O estudo do equilíbrio fornece informações fundamentais para avaliar a capacidade de diferentes adsorventes em adsorver um determinado adsorvato, sendo possível, desta forma, obter uma estimativa da quantidade máxima de contaminante a ser adsorvida [27].

A forma do gráfico da isoterma é determinada pelo mecanismo de adsorção e pode ser usada para sugerir o tipo de adsorção que ocorre entre o adsorvente e o adsorvato. Definir o perfil da isoterma é um ponto 
importante nos estudos relacionados ao processo de adsorção, já que elas não apresentam sempre o mesmo perfil, e dependem da natureza do adsorvente, do adsorvato e das condições do meio, como por exemplo, $\mathrm{pH}$, temperatura e outros. A classificação dada por GILES et al. [28] baseia-se nas inclinações iniciais e curvaturas das isotermas. Podem-se distinguir as isotermas de grande afinidade (H), Langmuir (L), constantes (C) e as de forma sigmóide (S) [28]. Observando os platôs, pontos de inflexão e máximos, as isotermas podem ser classificadas em subgrupos. Esta classificação é baseada na observação e não revela a ligação entre o processo e a forma da isoterma [29]. De forma geral, as isotermas do tipo $\mathrm{S}$ têm forma côncava a baixas concentrações. Enquanto as isotermas H e L têm forma convexa, a inclinação das isotermas $\mathrm{H}$ alcança valores altos e as isotermas $\mathrm{L}$ ficam constantes. Isto indica que a afinidade de sorção das isotermas $\mathrm{H}$ se incrementa com a diminuição da concentração. As isotermas tipo C são definidas pela afinidade de sorção constante, expressa por uma linha reta. Os subgrupos são definidos por seu comportamento a altas concentrações, subgrupo 1 não apresenta platôs, subgrupo 2 caracteriza-se por apresentar 1 platô, subgrupo 3 tem um ponto de inflexão devido à mudança para forma côncava. Dois platôs são característicos do subgrupo 4 [29].

De acordo com a classificação de GILES as isotermas para fibra de coco verde, in natura (Figura 9 A), tratada com hexano (Figura 9 B) e $\mathrm{HCl}$ (Figura 9 C), são do tipo L-3 indicando que os sítios ativos de adsorção diminuem sua disponibilidade quando a concentração da solução aumenta. Contudo, a partir de um valor de concentração mais elevado a adsorção aumenta à medida que o número de moléculas adsorvidas aumenta. A isoterma da fibra de coco verde tratada com NaOH (Figura 9 D) é do tipo L-1, indicando que os sítios ativos de adsorção diminuem sua disponibilidade quando a concentração da solução aumenta [28].
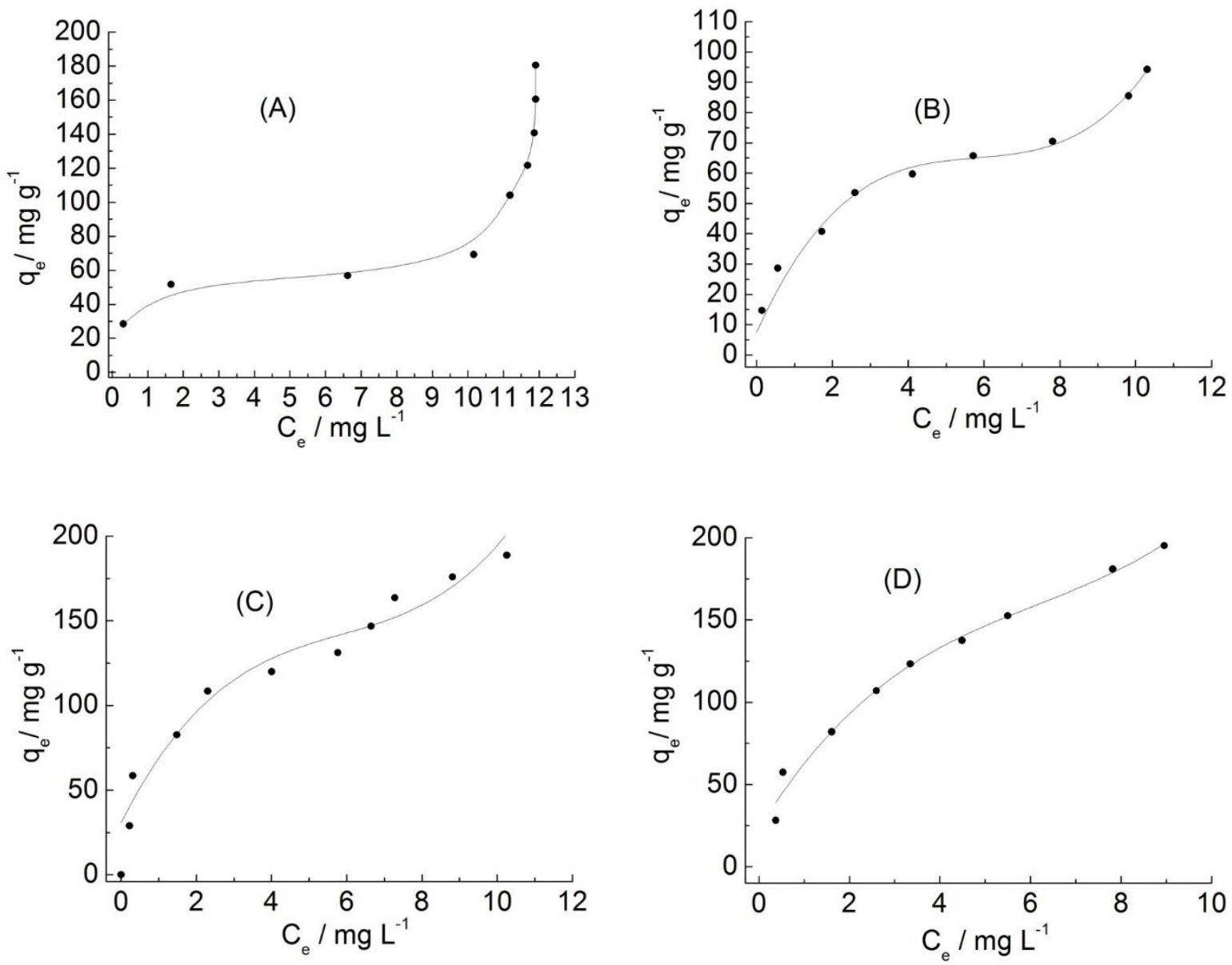

Figura 9: Isotermas de adsorção fibra de coco verde. In natura (A); tratada com: hexano (B); $\mathrm{HCl}(\mathrm{C}) ; \mathrm{NaOH}$ (D).

Os modelos de isotermas de Langmuir e Freundlich são os mais frequentemente utilizados para descrever o equilíbrio de adsorção, técnica aplicada em tratamento de águas e efluentes [30]. A isoterma de Langmuir é muito utilizada em inúmeros processos de adsorção principalmente na adsorção de corantes sobre superfícies sólidas. A equação de Langmuir é uma isoterma de equilíbrio baseada em um modelo teórico a qual relaciona a quantidade de soluto adsorvido em uma superfície com a concentração do soluto na solução. 
Este modelo é baseado na hipótese de que a máxima adsorção ocorre em uma monocamada saturada com moléculas de adsorvato na superfície do adsorvente que é energicamente homogênea contendo um número finito de sítios idênticos. A formação da monocamada depende da suposição que as forças intermoleculares diminuem com a distância e com isso só deve ocorrer uma única camada de soluto adsorvido. O modelo assume energias uniformes de sorção na superfície e que não há transmigração do adsorvato no plano da superfície. Segundo o modelo de Langmuir, a adsorção acontece em sítios específicos e igualmente disponíveis para a adsorção [31]. Todos os sítios são equivalentes e a superfície é uniforme, uma molécula se liga a um sítio independentemente dos demais estarem ocupados ou não. Teoricamente, o adsorvente tem capacidade finita pelo adsorvato [32]. A isoterma de Langmuir é representada pela equação 12.

$$
q_{e}=\frac{K_{L} \cdot C_{e q} \cdot q_{\max }}{1+K_{L} \cdot C_{e q}}
$$

Sendo: $\mathrm{q}_{\mathrm{e}}=$ quantidade adsorvida $\left(\mathrm{mg} \mathrm{g}^{-1}\right) ; \mathrm{q}_{\max }=$ quantidade máxima de adsorção $\left(\mathrm{mg} \mathrm{g}^{-1}\right) ; \mathrm{K}_{\mathrm{L}}=$ constante de equilíbrio de adsorção e indica a energia de ligação; $\mathrm{C}_{\mathrm{eq}}=$ concentração de equilíbrio $\left(\mathrm{mg} \mathrm{L}^{-1}\right)$.

Invertendo os termos da equação de Langmuir, temos a equação:

$$
\frac{1}{q_{e}}=\frac{1}{K_{L} q_{\max } C_{e q}}+\frac{1}{q_{\max }}
$$
14):

Multiplicando a equação 13 por $\mathrm{C}_{\mathrm{eq}}$, tem-se a forma linearizada para a equação de Langmuir (equação

$$
\frac{C_{e q}}{q_{e}}=\frac{1}{K_{L} q_{\max }}+\frac{C_{e q}}{q_{\max }}
$$

Considerando $\mathrm{C}_{\mathrm{e}} / \mathrm{q}_{\mathrm{e}}$ como variável dependente e $\mathrm{C}_{\mathrm{eq}}$ como variável independente, obtém-se os valores de $\mathrm{K}_{\mathrm{L}}$ e $\mathrm{q}_{\max }$, onde $1 /\left(\mathrm{K}_{\mathrm{L}} \mathrm{q}_{\max }\right)$ é o coeficiente linear e $1 / \mathrm{q}_{\max }$ é o coeficiente angular da reta. A partir dos parâmetros de adsorção obtidos pode-se avaliar a capacidade máxima de adsorção do adsorvato pelo adsorvente.

As características essenciais de uma isoterma de Langmuir podem ser expressas em termos de um fator de separação admissional constante ou comumente chamado parâmetro de equilíbrio, $\mathrm{R}_{\mathrm{L}}$, que é definido pela equação 15. O valor de $\mathrm{R}_{\mathrm{L}}$ indica o tipo de isoterma de adsorção, mostrado na Tabela 2 [33].

$$
R_{L}=\frac{1}{1+C_{0} \cdot K_{L}}
$$

Tabela 2: Classificação do tipo de isoterma de acordo com o parâmetro de equilíbrio.

\begin{tabular}{c|c}
\hline Parâmetro de equilíbrio & Tipo de isoterma \\
\hline $\mathrm{R}_{\mathrm{L}}>1$ & Desfavorável \\
\hline $\mathrm{R}_{\mathrm{L}}=1$ & Linear \\
\hline $0<\mathrm{R}_{\mathrm{L}}<1$ & Favorável \\
\hline $\mathrm{R}_{\mathrm{L}}=0$ & Irreversível \\
\hline
\end{tabular}

A isoterma de Freundlich é uma isoterma de adsorção empírica para uma adsorção não ideal em superfícies heterogêneas, bem como para uma adsorção em multicamada [34]. A isoterma de Freundlich é dada pela equação 16:

$$
q_{e}=K_{F} C_{e}^{1 / n}
$$

Sendo: $\mathrm{K}_{\mathrm{F}}=$ constante de Freundlich característica do sistema e indicadora da capacidade de adsorção; $\mathrm{n}=$ constante de Freundlich indicadora da intensidade de adsorção; $\mathrm{q}_{\mathrm{e}}=$ quantidade adsorvida $\left(\mathrm{mg} \mathrm{g}^{-1}\right)$

A forma linear da equação de Freundlich é dada pela equação 17:

$$
\log q_{e}=\log K_{F}+1 / n \log C_{e}
$$

Os valores de $\mathrm{K}_{\mathrm{F}}$ e n podem ser obtidos pela intersecção e inclinação do gráfico linear de $\log \mathrm{q}_{\mathrm{e}} \mathrm{vs} \log$ $\mathrm{C}_{\mathrm{e}}$. O valor de $\mathrm{n}$ entre 1 e 10 indica adsorção favorável.

Com os resultados experimentais obtidos calculou-se os parâmetros das isotermas de acordo com os 
modelos de Langmuir e Freundlich, afim de verificar se a isoterma experimental obtida segue o comportamento de alguns destes, pois apesar de os modelos empíricos não refletirem as questões relacionadas com o mecanismo da adsorção, eles fornecem informações úteis sobre a capacidade de acumulação de um adsorvente $[35]$.

O modelo de Langmuir considera a sorção em monocamada na superfície, com um número definido de lugares disponíveis. O modelo de Freundlich, por sua vez, considera que a sorção se dá em superfícies heterogêneas. Os parâmetros calculados estão apresentados na Tabela 3.

Tabela 3: Constantes de adsorção segundo os modelos de Langmuir e Freundlich para a remoção do azul de metileno utilizando fibra de coco verde in natura e tratada. Condições experimentais: $\mathrm{m} / \mathrm{v}$ : in natura $=200 \mathrm{mg} \mathrm{L}^{-1}$; hexano $=400$ $\mathrm{mg} \mathrm{L}^{-1} ; \mathrm{NaOH}=200 \mathrm{mg} \mathrm{L}^{-1} ; \mathrm{HCl}=200 \mathrm{mg} \mathrm{L}^{-1} ; \mathrm{pH}=8$; tempo de agitação: in natura= 60 min; hexano= 40min; $\mathrm{NaOH}=$ $60 \mathrm{~min} ; \mathrm{HCl}=60 \mathrm{~min}$. Temperatura ambiente.

\begin{tabular}{c|l|c|c|c|c}
\cline { 3 - 5 } \multicolumn{2}{c}{} & \multicolumn{1}{c}{ in natura } & hexano & HCl & NaOH \\
\hline \multirow{5}{*}{ Langmuir } & $\mathrm{q}_{\max }\left(\mathrm{mg} \mathrm{g}^{-1}\right)$ & 104,8 & 69,93 & 166,67 & 54,05 \\
\cline { 2 - 6 } & $\mathrm{K}_{\mathrm{L}}\left(\mathrm{L} \mathrm{mg}^{-1}\right)$ & 0,0000802 & 0,000114 & 0,00003 & 0,00075 \\
\cline { 2 - 6 } & $\mathrm{R}_{\mathrm{L}}$ & 0,999 & 0,998 & 0,9996 & 0,993 \\
\cline { 2 - 6 } & $\mathrm{r}$ & 0,89895 & 0,9569 & 0,8991 & 0,9292 \\
\hline \multirow{5}{*}{ Freundlich } & $\mathrm{N}$ & 0,18915 & 2,45 & 2,515 & 2,116 \\
\cline { 2 - 6 } & $1 / \mathrm{n}$ & 5,267 & 0,4074 & 0,3976 & 0,4725 \\
\cline { 2 - 6 } & $\mathrm{K}_{\mathrm{F}}\left(\mathrm{L} \mathrm{mg}^{-1}\right)$ & 0,0003146 & 33,65 & 71,79 & 16,83 \\
\cline { 2 - 6 } & $\mathrm{R}$ & 0,95247 & 0,9531 & 0,9579 & 0,9823 \\
\hline
\end{tabular}

Os valores das constantes de adsorção mostram que o modelo de Freundlich ajusta-se muito bem aos dados experimentais obtidos para a fibra de coco verde in natura e tratada com $\mathrm{HCl}$ e $\mathrm{NaOH}$, como pode ser observado pelos valores dos coeficientes de correlação, $\mathrm{R}$, apresentados (Tabela 3).

As constantes $K_{F}$ e 1/n são constantes de Freundlich. A constante $K_{F}$ é uma medida aproximada da capacidade de adsorção do adsorvente. Quanto maior o seu valor, maior é a capacidade de adsorção. A fibra de coco tratada com $\mathrm{HCl}$, apresentou valor de $\mathrm{K}_{\mathrm{F}}$ de $71,9 \mathrm{~L} \mathrm{mg}^{-1}$, ou seja, um aumento significativo na capacidade de adsorção, em relação a fibra de coco in natura e aos demais tratamentos.

Os valores de $n>1$ e $R_{L}\left(0<R_{L}<1\right)$ indicaram que o processo de adsorção foi favorável para a faixa de concentração estudada. A constante $1 / \mathrm{n}$ tem valor entre 0 e 1 e está relacionada à heterogeneidade da superfície. Quanto mais próxima de zero, mais heterogênea é a superfície. A fibra de coco verde tratada com $\mathrm{HCl}$ foi a que apresentou maior heterogeneidade, o tratamento promoveu um aumento da heterogeneidade quando comparada com o material in natura.

Os valores de $\mathrm{q}_{\max }$ obtidos pelo modelo de Langmuir para a fibra de coco in natura e tratada refletem elevada capacidade adsortiva, para azul de metileno, em relação a outros materiais adsorventes reportados na literatura, tais como, palmito pupunha $\left(\mathrm{q}_{\max }=50,96 \mathrm{mg} \mathrm{g}^{-1}\right)$ [36]; bagaço de cana-de-açúcar $\left(\mathrm{q}_{\max }=31,79 \mathrm{mg}\right.$ $\left.\mathrm{g}^{-1}\right)$ [37] e casca de maracujá $\left(\mathrm{q}_{\max }=44,70 \mathrm{mg} \mathrm{g}^{-1}\right)$ [38], sendo a fibra de coco tratada com $\mathrm{HCl}$ a que apresentou maior capacidade adsortiva $\left(166,67 \mathrm{mg} \mathrm{g}^{-1}\right)$, quando comparada aos demais tratamentos, in natura e tratados com $\mathrm{NaOH}$ e hexano.

\section{CONCLUSÕES}

A fibra de coco verde in natura e tratada com $\mathrm{HCl}, \mathrm{NaOH}$ e hexano foram propostas como materiais adsorventes aplicados na remoção de corante azul de metileno em soluções aquosas. A adsorção seguiu uma cinética de Pseudo segunda ordem e o modelo de adsorção de Freundlich, onde os valores das constantes, desse modelo, indicaram uma superfície mais heterogênea e um aumento na capacidade adsortiva para os materiais tratados, com destaque para a fibra de coco tratada com $\mathrm{HCl}$, cuja capacidade adsortiva foi de $166,7 \mathrm{mg} \mathrm{g}^{-1}$, sendo portanto, um material alternativo a ser utilizado na remoção do azul de metileno em efluentes da indústria têxtil por processo de adsorção. 


\section{BIBLIOGRAFIA}

[1] HAN, G., LIANG, C. Z., CHUNG, T. S., et al., "Combination of forward osmosis (FO) process with coagulation/flocculation (CF) for potential treatment of textile wastewater", Water Research, v. 91, pp. 361$370,2016$.

[2] ARAVIND, P., SUBRAMANYAN, V., FERRO, S., et al., "Eco-friendly and facile integrated biologicalcum-photo assisted electrooxidation process for degradation of textile wastewater", Water Research, v. 93, pp. 230-241, 2016.

[3] KUNZ, A., PERALTA-ZAMORA, P., MORAES, S. G., et al., "Novas tendências no tratamento de efluentes têxteis", Quimica Nova, v. 25, n. 1, pp. 78-82, 2002.

[4] CARDOSO, N. F., LIMA, E. C., PINTO, I. S., et al., "Application of cupuassu shell as biosorbent for the removal of textile dyes from aqueous solution", Journal of Environmental Management, v. 92, pp. 12371247, 2011.

[5] LUIZ DOTTO, G., VIEIRA, M. L. G., GONÇALVES, J. O., et al., "Remoção dos corantes azul brilhante, amarelo crepúsculo E amarelo tartrazina de soluções aquosas utilizando carvão ativado, terra ativada, terra diatomácea, quitina e quitosana: estudos de equilíbrio e termodinâmica", Quimica Nova, v. 34, n. 7, pp. 1193-1199, 2011.

[6] PINO, G. A. H., Biossorção de Metais Pesados Utilizando Pó da Casca de Coco Verde (Cocos nucifera), Dissertação de M.Sc., PPEM-PUC, Rio de Janeiro, RJ, Brasil, 2005.

[7] MARTINS, C. R., JESUS JUNIOR, L. A., "Evolução da produção de coco no Brasil e o comércio internacional: panorama 2010”, Documentos Embrapa, v. 164, p. 32, 2011.

[8] SIMONIN, J. P., "On the comparison of pseudo-first order and pseudo-second order rate laws in the modeling of adsorption kinetics”, Chemical Engineering Journal, v. 300, pp. 254-263, 2016.

[9] DEBRASSI, A., LARGURA, M. C. T., RODRIGUES, C. A., “Adsorção do corante vermelho congo por derivados da O-carboximetilquitosana hidrofobicamente modificados," Quimica Nova, v. 34, n. 5, pp. 764770, 2011.

[10] WEBER, W. J., MORRIS, J.C., "Kinetics of Adsorption on Carbon from Solution”, Journal of the Sanitary Engineering Division, v. 89, n. 2, pp. 31-60, 1963.

[11] OLIVEIRA, F. M., MELO, E. I., SOUSA, P. A. R., et al., "Avaliação do processo adsortivo utilizando bagaço de cana-de açúcar como material adsorvente na remoção do corante azul de metileno", In: STOPPA, M. H., PITUBA, J. J. C. (eds), Tecnologias em Pesquisa: Ciências Exatas e Biológicas, 1 ed., capítulo 2, São Paulo, Blucher, 2017.

[12] YANG, H., YAN, R., CHEN, H., et al.,"Characteristics of hemicellulose, cellulose and lignin pyrolysis", Fuel, v. 86, n. 12-13, pp. 1781-1788, 2007.

[13] CAO, Y., TAN, H., "Structural characterization of cellulose with enzymatic treatment", Journal of Molecular Structure, v. 705, n. 1-3, pp. 189-193, 2004.

[14] ALVES, R. E., Caracterização de fibras lignocelulósicas pré-tratadas por meio de técnicas espectroscópicas e microscópicas ópticas de alta resolução, Dissertação de M.Sc., Universidade de São Paulo, São Carlos, 2011.

[15] HOAREAU, W., TRINDADE, W. G., SIEGMUND, B., et al., "Sugar cane bagasse and curaua lignins oxidized by chlorine dioxide and reacted with furfuryl alcohol: Characterization and stability", Polymer Degradation and Stability, v. 86, n. 3, pp. 567-576, 2004.

[16] GUPTA, M., MADAN, S., BANSAL, R.N., "Chemical composition of Pinus caribaea hemicelluloses", Tappi Journals, v. 70, pp. 14-113, 1987.

[17] MOHANTY, S., NAYAK, S. K., "Effect of MAPP as coupling agent on the performance of sisal-PP composites", Journal Reinforced Plastics and Composites, v. 23, n. 18, pp. 2047-2063, 2004.

[18] ALMEIDA, J. R. M. D., AQUINO, R. C. M. P., MONTEIRO, S. N., "Tensile mechanical properties, morphological aspects and chemical characterization (Attalea funifera) fibers", Composites Part A: Applied Science and Manufacturing, v. 37, n. 9, pp. 1473-1479, 2006.

[19] ESMERALDO, M. A., Preparação de novos compósitos suportados em matrizes de fibras vegetais, 2006. 99 f. Dissertação M.Sc., Universidade Federal do Ceará, Fortaleza, 2006.

[20] RATTA, V., Thesis on the crystallization, morphology, thermal stability and adhesive properties of nov- 
el high performance semicrystalline polyimides, Faculty of Virginia Polytechnic Institute and State University, Virginia, 1999.

[21] GAÑAN, P., MONDRAGON, I., "Effect of fiber treatments on mechanical behavior of short fique fiber-reinforced polyacetal composites", Journal of Composite Materials, v. 39, n. 7, pp. 633-646, 2005.

[22] MALL, I. D., SRIVASTAVA, V. C., AGARWAL, N. K., et al., "Removal of congo red from aqueous solution by bagasse fly ash and activated carbon: Kinetic study and equilibrium isotherm analyses", Chemosphere, v. 61, n. 4, pp. 492-501, 2005.

[23] DOTTO, G. L., PINTO,L. A. A., "Adsorption of food dyes acid blue 9 and food yellow 3 onto chitosan: Stirring rate effect in kinetics and mechanism", Journal of Hazardous Materials, v. 187, n. 1-3, pp. 164-170, 2011.

[24] JACQUES, R. A. , LIMA,E. C. , DIAS, S. L. P., et al., "Yellow passion-fruit shell as biosorbent to remove $\mathrm{Cr}(\mathrm{III})$ and $\mathrm{Pb}(\mathrm{II})$ from aqueous solution", Separation and Purification Technology, v. 57, n. 1, pp. 193-198, 2007.

[25] CARDOSO, N. F., et al., "Application of cupuassu shell as biosorbent for the removal of textile dyes from aqueous solution”, Journal of Environmental Management, v. 92, n. 4, pp. 1237-1247, 2011.

[26] CALVETE, T. , LIMA, E. C., CARDOSO, N. F., et al., "Application of carbon adsorbents prepared from Brazilian-pine fruit shell for the removal of reactive orange 16 from aqueous solution: Kinetic, equilibrium, and thermodynamic studies", Journal of Environmental Management, v. 91, n. 8, pp. 1695-1706, 2010.

[27] ROOSTAEI, N., TEZEL, F. H., "Removal of phenol from aqueous solutions by adsorption," Journal of Environmental Management, vol. 70, no. 2, pp. 157-164, 2004.

[28] GILES, S. N., MACEWAN, C.H., NAKHWA, T.H., "Studies in adsorption. Part XI.* A system of classification of solution adsorption isotherms, and its use in diagnosis of adsorption mechanisms and in measurement of specific surface areas of solids," Journal of the Chemical Society, vol. 1, no. 0, pp. 3973-3993, 1960.

[29] HINZ, C., "Description of sorption data with isotherm equations", Geoderma, v. 99, n. 3-4, pp. 225243, 2001.

[30] FUNGARO, D. A., IZIDORO, J. C., BRUNO, M., "Aplicação de material zeolítico sintetizado de cinzas de carvão como adsorvente de poluentes em água”, Ecletica Quimica, v. 34, n. 1, pp. 45-50, 2009.

[31] WALKER, G. M., WEATHERLEY, L. R., "Adsorption of dyes from aqueous solution - The effect of adsorbent pore size distribution and dye aggregation", Chemical Engineering Journal, v. 83, n. 3, pp. 201206, 2001.

[32] ÖZCAN, A. S., ÖZCAN, A., "Adsorption of acid dyes from aqueous solutions onto acid-activated bentonite", Journal of Colloid and Interface Science, v. 276, n. 1, pp. 39-46, 2004.

[33] VASQUES A.R., Caracterização e Aplicação de Adsorvente para Remoção de Corantes de Efluentes Têxteis em Batelada e Colunas de Leito Fixo, Dissertação de M.Sc., Universidade Federal de Santa Catarina, Florianópolis, 2008.

[34] AKSU, Z., TEZER, S., "Biosorption of reactive dyes on the green alga Chlorella vulgaris", Process Biochem., v. 40, n. 3-4, pp. 1347-1361, 2005.

[35] DO DUONG, D., Adsorption Analysis: Equilibria and Kinetic, 3 ed. London, 1998.

[36] HONORATO, A.C., MACHADO, J.C., CELANTE, G. et al., "Biossorção de azul de metileno utilizando resíduos agroindustriais", Revista Brasileira de Engenharia Agrícola e Ambiental, Campina Grande- PB, v. 19, n. 7, pp. 705-710, 2015.

[37] SILVA, W. L. L. D., OLIVEIRA, S. P. D., "Modificação das características de adsorção do bagaço de cana para remoção de azul de metileno de soluções aquosas”, Scientia Plena, v. 8, pp. 1-9, 2012.

[38] PAVAN, F. A., DIAS, S. L. P., MAZZOCATO, A. C., "Methylene blue biosorption from aqueous solutions by yellow passion fruit waste", Journal of Hazardous Materials, v.150, n. 3, pp. 703-712, 2008. 